\title{
Exact broken-symmetry states and Hartree-Fock solutions for quantum dots at high magnetic fields
}

\author{
B. Szafran ${ }^{\mathrm{a}, \mathrm{b}}$, F.M. Peeters ${ }^{\mathrm{a}}$, S. Bednarek ${ }^{\mathrm{b}}$, and \\ J. Adamowski ${ }^{\mathrm{b}}$ \\ ${ }^{a}$ Departement Natuurkunde, Universiteit Antwerpen (Campus drie Eiken), B-2610 \\ Antwerpen, Belgium \\ ${ }^{\mathrm{b}}$ Faculty of Physics and Nuclear Techniques, AGH University of Science and \\ Technology, Kraków, Poland
}

\begin{abstract}
Wigner molecules formed at high magnetic fields in circular and elliptic quantum dots are studied by exact diagonalization (ED) and unrestricted Hartree-Fock (UHF) methods with multicenter basis of displaced lowest Landau level wave functions. The broken symmetry states with semi-classical charge density constructed from superpositions of the ED solutions are compared to the UHF results. UHF overlooks the dependence of the few-electron wave function on the actual relative positions of electrons localized in different charge puddles and partially compensates for this neglect by an exaggerated separation of charge islands which are more strongly localized than in the exact broken-symmetry states.
\end{abstract}

Key words: quantum dots, Wigner molecules, PACS: 73.21.La, 73.20.Qt 
At high magnetic fields the electron systems in circular quantum dots form Wigner molecules $[1,2]$ in the internal structure of the system. Deformation $[3,4]$ of the circular symmetry allows the molecules to appear in the laboratory frame only [4] when the classical counterpart [5] of the quantum system possesses a single lowest-energy configuration. Otherwise the charge density at high field is a superposition of equivalent semi-classical densities and the quantum system undergoes symmetry transformations when the magnetic field is increased [4]. These transformations are associated with level crossings at which the ground state is two-fold degenerate. Superposition of the states of the degenerate levels allows [4] to extract the semi-classical brokensymmetry charge density into the laboratory frame. On the other hand the unrestricted Hartree-Fock (UHF) produces [1] broken-symmetry states for Wigner molecules. In the infinite magnetic field limit UHF gives exact [6] results for the total energy. At finite magnetic fields for which exact brokensymmetry eigenstates exist the artifactal symmetry breaking cannot be blamed to the inaccuracy of the UHF [4]. In this paper we look for the effects neglected by UHF comparing the ED and UHF solutions for elliptical and circular dots.

We assume a spin-polarization of electrons at high magnetic field $(0,0, B)$ oriented perpendicular to the quantum dot plane and use the Landau gauge. In the ED, described in detail in Ref. [4], the single electron wave functions used for construction of the Slater determinants are obtained via diagonalization of the single electron Hamiltonian in the multicenter basis [7,8,9] of $M$ displaced lowest Landau level wave functions

$$
\begin{gathered}
\psi(\mathbf{r})=\sum_{k=1}^{M} c_{k} \exp \left\{-\alpha\left[\left(x-x_{k}\right)^{2}+\left(y-y_{k}\right)^{2}\right] / 4+\right. \\
\left.+i e B\left(x-x_{k}\right)\left(y+y_{k}\right) / 2 \hbar\right\}
\end{gathered}
$$

where $\alpha$ is treated as variational parameter. In the present UHF approach the one-electron orbitals (1) are optimized self-consistently. We study up to $N=4$ electrons, use the material data of GaAs and a basis of 12 centers $\left(x_{k}, y_{k}\right)$ put on an ellipse with a size determined variationally. Basis (1) of displaced lowest Landau level wave functions reproduces [4] also higher Fock-Darwin bands. Contrary to previous multicenter HF calculations [6,9] using a single center per electron, the present $\mathrm{HF}$ approach produces results which are exact in the UHF [10] sense.

Classical system of three electrons in an elliptical confinement potential with $\hbar \omega_{x}=3 \mathrm{meV}$ and $\hbar \omega_{y}=4 \mathrm{meV}$ possesses two equivalent lowest-energy configurations [cf. inset of Fig. 1] and the quantum system undergoes parity transformations [4] with the magnetic field [cf. Fig. 1]. Superposition [4] of the two lowest-energy eigenstates

$$
\Psi_{B S}=\left(\Psi_{\text {even }}+e^{i \phi} \Psi_{\text {odd }}\right) / \sqrt{2}
$$


yields a broken-symmetry (BS) charge density with a distinct electron separation. Fig. 1 shows that in contrast to the exact ground-state energy the UHF energy estimate is a smooth function of the magnetic field.

The charge densities of considered states are shown in Figs. 2(a) and 2(b) for two magnetic field values corresponding to the even-odd energy crossing presented in Fig. 1. The phase $\phi$ in the BS state is chosen such that the electrons are localized at the classical Wigner molecule positions. Notice that in the UHF the separation of electrons is more pronounced than in the exact BS states. Fig. 2(c) shows the pair correlation function (PCF) [2] for the UHF and the exact BS state corresponding to the charge density of Fig. 2(a) with the position of one of the electrons fixed at two different locations: in the center and on the edge of the central charge puddle. In contrast to the exact BS state in the UHF wave function the two electrons are insensitive to the actual position of the third electron in its charge puddle. This is a consequence of the single-determinantal form of the UHF wave function, and can be easily explained for two electrons. In the spin-polarized two electron Wigner molecule the UHF spatial wave function is given by $\Psi_{\alpha}\left(\mathbf{r}_{1}\right) \Psi_{\beta}\left(\mathbf{r}_{2}\right)-\Psi_{\beta}\left(\mathbf{r}_{1}\right) \Psi_{\alpha}\left(\mathbf{r}_{2}\right)$, where $\left|\Psi_{\alpha}\right|^{2}$ and $\left|\Psi_{\beta}\right|^{2}$ are the charge densities of separate charge puddles $\alpha$ and $\beta$. Wave functions $\Psi_{\alpha}$ and $\Psi_{\beta}$ are orthogonal due to the vanishing overlap between the puddles. The calculation of the PCF gives (up to a constant) $\operatorname{PCF}\left(\mathbf{r}_{a}, \mathbf{r}_{b}\right)=\left|\Psi_{\alpha}\left(\mathbf{r}_{a}\right)\right|^{2}\left|\Psi_{\beta}\left(\mathbf{r}_{b}\right)\right|^{2}+\left|\Psi_{\alpha}\left(\mathbf{r}_{b}\right)\right|^{2}\left|\Psi_{\beta}\left(\mathbf{r}_{a}\right)\right|^{2}$. For $\mathbf{r}_{b}$ inside puddle $\beta$ the second term of the sum vanishes and the remaining one signifies that the probability of finding an electron in point $\mathbf{r}_{a}$ inside puddle $\alpha$ is independent of the actual position of the second electron in puddle $\beta$.

The UHF self-consistency is reached only in one of the two classical orientations [cf. inset of Fig. 1] in which the UHF energy is minimal. On the other hand, the exact BS states can be oriented under an arbitrary angle [cf. Fig. 2(d)] by modifying the phase $\phi$ in Eq. (2). Moreover, since the BS state is constructed with states of opposite parities all the plots in Fig. 2(d) correspond to the same value of the kinetic, potential and electron-electron interaction energies equal to the arithmetic average of the expectation values for $\Psi_{\text {odd }}$ and $\Psi_{\text {even }}$ states.

Fig. 3 shows the two lowest energy levels and the UHF total energy calculated with respect to the lowest Landau level for the elliptical dot with $\hbar \omega_{x}=3$ $\mathrm{meV}$ and $\hbar \omega_{y}=4 \mathrm{meV}$. For these values the classical counterpart of the fourelectron system is unique and conform with the symmetry of the confinement potential, so that the Wigner crystallization is visible in the exact quantum ground-state for an arbitrary magnetic field after the MDD decay. In this case the MDD decay is a continuous process and appears at the anticrossing around $6 \mathrm{~T}$.

The inset to Fig. 3 shows the charge densities calculated with the UHF and 
ED methods. In ED the charge density in between the charge maxima takes on larger values than in UHF in which the separation of electrons is more distinct like for $N=3$ [cf. Fig. 2(a) and (b)].

The average radius of the charge puddle as obtained for the two-electron ground state in the circular quantum dot $\left(\hbar \omega_{x}=\hbar \omega_{y}=3 \mathrm{meV}\right)$ is displayed in Fig. 4. The ED and UHF values are similar below the MDD breakdown $(B<5.6 \mathrm{~T})$. The exact value present discontinuities at the angular momentum transitions. After the MDD decay the UHF value is close to the average around which ED and BS results oscillate, but at higher fields it becomes an upper bound for these oscillations. The inset of Fig. 4 shows the exact BS and UHF charge density for $B=11.8$ and $28.9 \mathrm{~T}$. BS charge densities for the two values of the magnetic field have been obtained from superpositions of the degenerate states with angular momenta $-5,-7$ and $-9,-11$ (in $\hbar$ units), respectively. The charge maxima in the exact $\mathrm{BS}$ solutions are less strongly separated. For $B=28.9 \mathrm{~T}$ the charge density islands of the exact BS have crescent shape while the UHF charge puddles are more oval and the distance between them is larger.

The difference in shape of the separated charge islands in circular dots is largest for $N=2$. For larger $N$ the charge puddles in the exact solutions are less spectacularly spread. Fig. 5 shows the comparison of the exact BS and UHF charge densities for $N=3$. The BS plots for $N=3$ correspond to degeneracy of states with angular momentum $-9 \hbar,-12 \hbar$ for $B=7.5 \mathrm{~T}$ and $-12 \hbar,-15 \hbar$ for $B=15 \mathrm{~T}$.

In summary, we have presented a comparison of the UHF and the ED results for the charge density of Wigner molecules in circular and elliptical dots. For the comparison we have used the broken-symmetry states obtained from the superposition of the exact eigenstates. We have found that the UHF exaggerates the separation of the electron charge densities in the laboratory frame. In this way the UHF method partially compensates for the overlooked correlations related to the reaction of electrons on their actual position in the separated charge density islands. This reaction is of smaller importance for larger magnetic fields, for which the charge density islands shrink to points, which explains the vanishing of the UHF energy overestimation in the infinite magnetic field [6]. Due to the exaggerated electron separation the charge islands forming Wigner molecules calculated in the UHF shrink with the magnetic field faster than in the exact broken-symmetry states.

Acknowledgments This paper was supported in part by the Polish Ministry of Scientific Research and Information Technology in the framework of the solicited grant PBZ-MIN-008/P03/2003, the Flemish Science Foundation (FWO-Vl), the Belgian Science Policy, and the University of Antwerpen (VIS and GOA). One of us (BS) is supported by the Foundation for Polish Science 
(FNP).

\section{References}

[1] S.M. Reimann and M. Manninen, Rev. Mod. Phys. 74 (2002) 1283.

[2] P.A. Maksym, H. Immamura, G.P. Mallon, and H. Aoki, J. Phys. Condens. Matter 12 (2000) R299.

[3] M. Manninen, M. Koskinen, S.M. Reimann, and B. Mottelson, Eur. Phys. J. D. 16 (2001) 381.

[4] B. Szafran, F.M. Peeters, S. Bednarek, and J. Adamowski, Phys. Rev. B 69 (2004) 125344.

[5] V.M. Bedanov and F.M. Peeters, Phys. Rev. B 49 (1994) 2667.

[6] B. Szafran, S. Bednarek, J. Adamowski, M. Tavernier, E. Anisimovas, and F.M. Peeters, Eur. Phys. J. D 28 (2004) 373.

[7] J. Kainz, S.A. Mikhailov, A. Wensauer, and U. Rössler, Phys. Rev. B 65 (2002) 115305 .

[8] C. Yannouleas and U. Landman, Phys. Rev. B 66 (2002) 115315.

[9] B. Szafran, S. Bednarek, and J. Adamowski, Phys. Rev. B 67 (2003) 045311; J. Phys.: Cond. Matter 15 (2003) 4189.

[10] B. Reusch and H. Grabert, Phys. Rev. B 68 (2003) 045309.

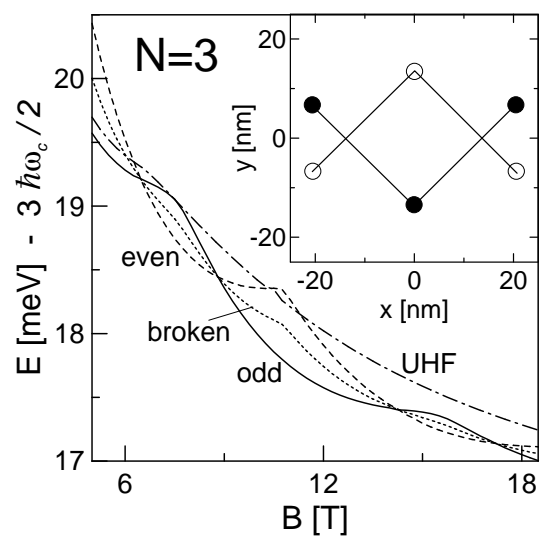

Fig. 1. Energy of the lowest even (dashed curve) and odd parity (solid curve) levels, the broken symmetry state (dotted curve) and UHF energy calculated for $N=3$, $\hbar \omega_{x}=3 \mathrm{meV}$ and $\hbar \omega_{y}=4 \mathrm{meV}$. Inset shows the two equivalent classical configurations. 


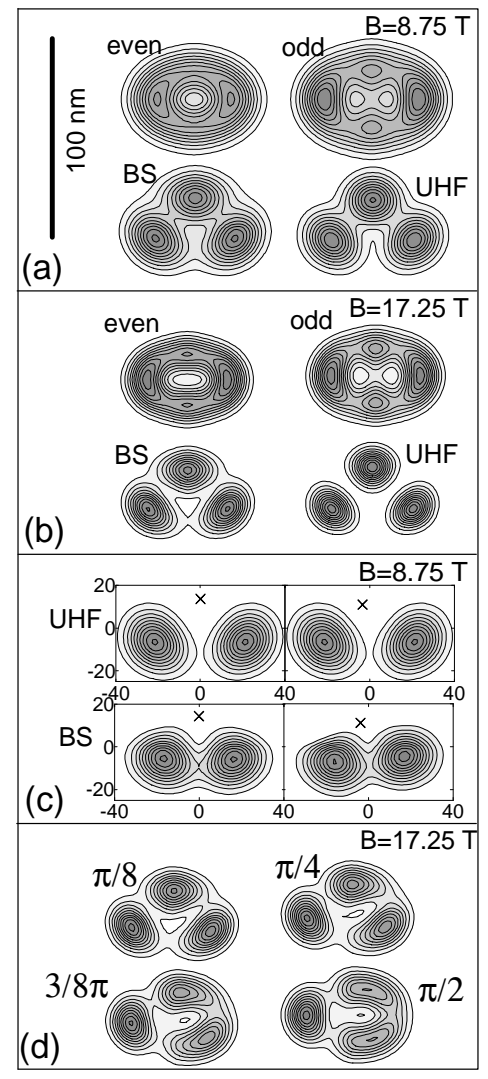

Fig. 2. (a) The charge densities for the even, the odd, the broken-symmetry (BS) state and the UHF for $N=3, \hbar \omega_{x}=3 \mathrm{meV}, \hbar \omega_{y}=4 \mathrm{meV}$ at $B=8.75 \mathrm{~T}$. (b) Same as (a) at $B=17.25 \mathrm{~T}$. (c) Pair correlation function plots for UHF and BS state for an electron fixed at $(0,13.4 \mathrm{~nm})$ [left panel] and $(-3 \mathrm{~nm}, 10.4 \mathrm{~nm})$ [right panel] marked by crosses. (d) BS states for given shifts of phase $\phi$ [Eq. (2)] with respect to the BS plot in (b). Plots (a), (b) and (d) have the same scale given by the length bar in (a). 


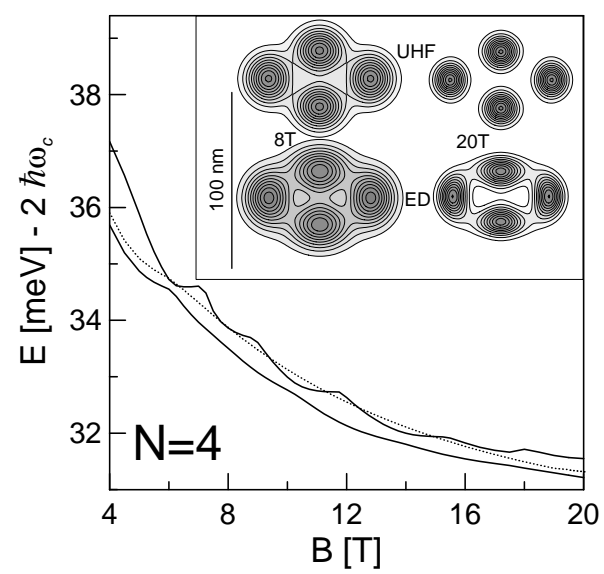

Fig. 3. Lowest energy levels for $N=4, \hbar \omega_{x}=3 \mathrm{meV}$ and $\hbar \omega_{y}=4 \mathrm{meV}$ (solid lines) and total UHF energy (dotted) line. Inset: Charge density calculated in the UHF (two upper plots) and the ED (two lower plots) for $B=8 \mathrm{~T}$ (left side) and $B=20 \mathrm{~T}$ (right side).

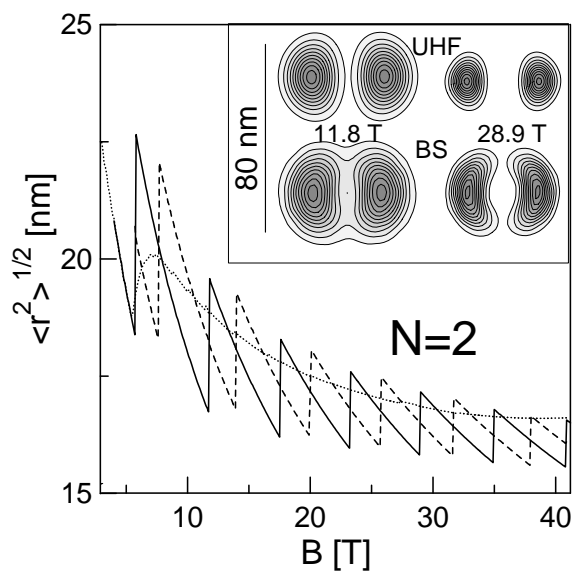

Fig. 4. Average radius of the charge puddle $\left(<r^{2}>^{1 / 2}\right)$ for two electrons in a circular quantum dot $\left(\hbar \omega_{x}=\hbar \omega_{y}=3 \mathrm{meV}\right)$. Dotted, dashed and solid lines correspond to UHF, BS, and ED results. Inset: charge density obtained in the UHF (two upper plots) and in the exact broken-symmetry state (two lower plots) for $\mathrm{B}=11.8 \mathrm{~T}$ (left side) and $28.9 \mathrm{~T}$ (right side). 


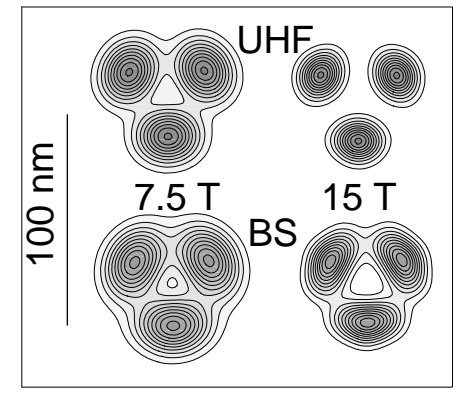

Fig. 5. Charge density for $N=3$ electrons in a circular quantum dot $\left(\hbar \omega_{x}=\hbar \omega_{y}=3 \mathrm{meV}\right.$ ) calculated with UHF (upper plots) and for exact BS states (lower plots) for $B=7.5 \mathrm{~T}$ (left plots) and $B=15 \mathrm{~T}$ (right plots). 